\title{
Approximate and weak amenability of certain Banach algebras
}

\author{
by \\ P. Bharucha and R. J. LoY (Canberra)
}

\begin{abstract}
The notions of approximate amenability and weak amenability in Banach algebras are formally stronger than that of approximate weak amenability. We demonstrate an example confirming that approximate weak amenability is indeed actually weaker than either approximate or weak amenability themselves. As a consequence, we examine the (failure of) approximate amenability for $\ell^{p}$-sums of finite-dimensional normed algebras.
\end{abstract}

1. Introduction. Various notions of amenability in Banach algebras have surfaced over the years which attempt to make sharp the tradeoff between the restrictiveness of the notion (that is, how many examples of Banach algebras satisfy it) and the hereditary theory associated with it. This effort has led to two distinct approaches - one being to restrict the bimodules in question, yielding such notions as weak amenability; and the other being to somewhat relax the structure on the derivations themselves yielding such notions as approximate amenability [9].

Definition 1.1. A Banach algebra $A$ is weakly amenable if each continuous derivation $D: A \rightarrow A^{*}$ is inner.

Definition 1.2. A Banach algebra $A$ is approximately amenable if, for each Banach $A$-bimodule $E$ and every continuous derivation $D: A \rightarrow E^{*}$, there exists a net $\left(x_{\alpha}^{*}\right) \subset E^{*}$ such that $D$ is given by

$$
D(a)=\lim _{\alpha}\left(a \cdot x_{\alpha}^{*}-x_{\alpha}^{*} \cdot a\right) \quad(a \in A) .
$$

We examine the combination of these two notions.

Definition 1.3. A Banach algebra $A$ is approximately weakly amenable if for every continuous derivation $D: A \rightarrow A^{*}$ there is a net $\left(a_{\alpha}^{*}\right) \subset A^{*}$ such

2010 Mathematics Subject Classification: Primary 46H20; Secondary 43A15, 43A32.

Key words and phrases: amenability, approximate amenability, weak amenability, Segal algebras. 
that

$$
D(a)=\lim _{\alpha}\left(a \cdot a_{\alpha}^{*}-a_{\alpha}^{*} \cdot a\right) \quad(a \in A) .
$$

Given what has already been said, this is a natural combination of the two distinct methods for generalizing amenability. Approximate weak amenability has not been extensively studied, except for Segal algebras [7. One of our main goals is to remedy this situation. Until now it has not been established that approximate weak amenability is totally independent of weak amenability, or of approximate amenability. The broad purpose of this paper is to demonstrate this independence. To be specific, we seek an example of a Banach algebra which is approximately weakly amenable but which fails to be approximately amenable or weakly amenable.

2. The example. Via a very simple argument, we can immediately rule out a large class of Banach algebras from our search.

Proposition 2.1. Suppose that $A$ is a commutative Banach algebra which is approximately weakly amenable. Then $A$ is weakly amenable.

Proof. Consider a continuous derivation $D: A \rightarrow A^{*}$. Then by hypothesis there is a net $\left(x_{\alpha}^{*}\right) \subset A^{*}$ such that for each $a \in A$,

$$
D(a)=\lim _{\alpha}\left(a \cdot x_{\alpha}^{*}-x_{\alpha}^{*} \cdot a\right)=0,
$$

since $A^{*}$ is a commutative $A$-bimodule.

That is to say, for commutative Banach algebras, approximate weak amenability and weak amenability are the same.

As previously stated, the only concerted effort to study approximate weak amenability has been carried out on Segal algebras. We recall the basic definitions involved. Given a function $f$ defined on a group $G$, we denote the left and right translations of $f$ by $x \in G$ as

$$
l_{x} f(y)=f(x y), \quad r_{x}(y)=f(y x) \quad(y \in G) .
$$

Definition 2.2. A Segal algebra on a locally compact group $G$ is a linear subspace $S(G)$ of the convolution algebra $L^{1}(G)$ which satisfies the following properties:

- $S(G)$ is dense in $L^{1}(G)$,

- $S(G)$ is a Banach space under some norm $\|\cdot\|_{S}$ and $\|f\|_{S} \geq\|f\|_{1}$ for all $f \in S(G)$,

- $S(G)$ is left-translation invariant, and $\left\|l_{x} f\right\|_{S}=\|f\|_{S}$ for every $x \in G$ and $f \in S(G)$, and the map $x \mapsto l_{x} f$ from $G$ into $S(G)$ is continuous.

Under these properties, it is standard that a Segal algebra is indeed a Banach algebra, contained as an ideal in $L^{1}(G)$. A Segal algebra is symmetric if the analogous statement also holds for right translations. 
To use what is known about approximate weak amenability on Segal algebras, we also need to slightly restrict the class of groups considered.

Definition 2.3. A locally compact group $G$ is called a $S I N$ group if there is a basis for neighbourhoods of the identity of $G$ consisting of compact sets $U_{\alpha}$ for which $x U_{\alpha} x^{-1}=U_{\alpha}$ for all $\alpha$ and $x \in G$.

It is standard that many commonly considered groups are of class SIN, for example all compact groups, discrete groups and abelian groups.

The main result obtained for Segal algebras is the following. We see that many of the most common Segal algebras are in fact approximately weakly amenable.

Theorem 2.4 ([7, Theorem 2.1]). Suppose that $S(G)$ is a symmetric Segal algebra on a SIN group. Then $S(G)$ is approximately weakly amenable.

There is also a result of this type for weak amenability [8]. However, the fact is that it assumes commutativity of the Segal algebra, that is, $G$ must be abelian (this result is, of course, obvious from Theorem 2.4 in light of the equivalence of weak and approximate weak amenability under the commutativity hypothesis). A partial converse to this is the following observation. Note that $L^{2}(G)$ is, of course, a symmetric Segal algebra on $G$ whenever $G$ is compact.

Proposition 2.5 ([8, Remark 3.2]). Suppose that $G$ is a compact nonabelian group. Then $L^{2}(G)$ is not weakly amenable.

This indicates the possibility that there might be a compact non-abelian group $G$ such that $L^{2}(G)$ fulfils the required criteria. We look for a nonabelian compact group $G$ such that $L^{2}(G)$ is not approximately amenable. Clearly, this group must be infinite.

To determine a possible approach for this, one looks to the abelian case, even though this is not what is ultimately desired. We have already chosen to investigate the case of compact $G$. Suppose then for the moment that $G$ is abelian. Then the standard Fourier transform gives a Banach algebra isomorphism of $L^{2}(G)$ onto $\ell^{2}(\widehat{G})$ under pointwise multiplication. We thus need a result relating to the approximate amenability of $\ell^{2}$-sums.

Lemma 2.6. For $1 \leq p<\infty$ and any infinite set $I, \ell^{p}(I)$ fails to be approximately amenable.

This is the observation directly following Theorem 4.1 of [5]. Thus $\ell^{2}(\widehat{G})$ fails approximate amenability, and hence $L^{2}(G)$ fails approximate amenability.

Motivated by this we turn to the representation theory of compact groups. The reader is referred to [6] and [10] for further details. For $G$ 
a locally compact group, the dual object of $G$, denoted by $\Sigma=\Sigma(G)$, is the collection of equivalence classes of irreducible unitary representations of $G$.

Definition 2.7 ([10,28.24]). Let $I$ be an index set. For each $i \in I$, let $H_{i}$ be a Hilbert space of dimension $d_{i}$. Then denote $\bigoplus_{i \in I} B\left(H_{i}\right)$ by $\mathfrak{G}(I)$. Define a Hilbert space direct sum norm on $\mathfrak{G}(I)$ by

$$
\|E\|_{2}=\left(\sum_{i \in I} d_{i}\left\|E_{i}\right\|_{\mathrm{tr}}^{2}\right)^{1 / 2}
$$

and corresponding space $\mathfrak{G}_{2}(I)=\left\{E \in \mathfrak{G}(I):\|E\|_{2}<\infty\right\}$; here, $\|M\|_{\text {tr }}$ denotes the trace class norm of a matrix $M$.

It is shown in [10, Corollary 28.29] that $\mathfrak{G}_{2}(I)$ is a Hilbert space under the inner product

$$
\langle E, F\rangle=\sum_{i \in I} d_{i} \operatorname{tr}\left(E_{i} F_{i}^{*}\right),
$$

thus generalizing the Hilbert space $\ell^{2}$.

One now considers the index set $\Sigma$, with $d_{\sigma}=\operatorname{dim} H_{\sigma}$ for $\sigma \in \Sigma$, and uses coordinatewise product on $\mathfrak{G}_{2}(\Sigma)$ to turn it into a Banach algebra. Note that if $G$ were abelian, then $\mathfrak{G}_{2}(\Sigma)$ would correspond to $\ell^{2}(\Sigma)$ both in terms of topology and multiplication. We now make use of the algebra $\mathfrak{G}_{2}(\Sigma)$; it puts us in a position to define an appropriate Fourier transform.

It should be noted that if one uses [10, Definitions 28.34] to define our Fourier transform $f \mapsto \widehat{f}$ for $f \in L^{1}(G)$, it is given in terms of the decompositions of the unitary representations of $G$. We use an equivalent definition, which is in the notation of [6, p. 134], circumventing the need to specify bases for the Hilbert spaces involved.

Definition 2.8. The Fourier transform of $f \in L^{1}(G)$ at $\sigma \in \Sigma$ is the operator

$$
\widehat{f}(\sigma)=\int f(x) \sigma(x) d h(x),
$$

which maps onto a dense subset of $c_{0}(\Sigma)=\left\{E \in \mathfrak{G}(I):\left\|E_{i}\right\|_{\operatorname{tr}} \stackrel{i}{\rightarrow} 0\right\}$. As usual, $h$ is the unique normalized Haar measure on the group $G$.

To make sense of this definition, one should view it in the weak sense. That is, for any $u \in H_{\sigma}$ we define $\widehat{f}(\sigma) u$ by specifying its inner product with another arbitrary $v \in H_{\sigma}$, and this is given by

$$
\langle\widehat{f}(\sigma) u, v\rangle=\int f(x)\langle\sigma(x) u, v\rangle d h(x) .
$$

Then this Fourier transform has the desired properties.

Theorem 2.9 ([10, Theorems 28.40 and 28.43]). Suppose that $G$ is a compact group. Then the transform $f \mapsto \widehat{f}$ maps $L^{2}(G)$ isometrically onto 
$\mathfrak{G}_{2}(\Sigma)$. Also, this map is an isomorphism, in that $\widehat{f * g}(\sigma)=\widehat{f}(\sigma) \widehat{g}(\sigma)$ for $f, g \in L^{2}(G)$ and $\sigma \in \Sigma$.

Hence the Fourier transform is actually a Banach algebra isomorphism from $L^{2}(G)$ onto $\mathfrak{G}_{2}(\Sigma)$. This means that if we can establish that $\mathfrak{G}_{2}(\Sigma)$ fails to be approximately amenable, then $L^{2}(G)$ will also fail to be approximately amenable.

Let us for the moment assume that the group has infinitely many onedimensional representations, somewhat resembling the abelian case. Write $\Sigma_{1}=\left\{\sigma \in \Sigma: \operatorname{dim} H_{\sigma}=1\right\}$. Then

$$
\mathfrak{G}_{2}(\Sigma)=\mathfrak{G}_{2}\left(\Sigma_{1}\right) \oplus \mathfrak{G}_{2}\left(\Sigma \backslash \Sigma_{1}\right),
$$

with both of these summands being ideals; recall that we are using coordinatewise product. Since the product on $\mathfrak{G}_{2}\left(\Sigma_{1}\right)$ coincides with the pointwise product on $\ell^{2}\left(\Sigma_{1}\right)$, and denoting $I=\mathfrak{G}_{2}\left(\Sigma \backslash \Sigma_{1}\right)$, we have

$$
\mathfrak{G}_{2}(\Sigma) / I=\ell^{2}\left(\Sigma_{1}\right) \text {. }
$$

Note that when we write $\ell^{2}\left(\Sigma_{1}\right)$, it is clear that we are referring to complexvalued sequences over the index set $\Sigma_{1}$. However, we will soon encounter $\ell^{p}(A)$, where $A$ is a Banach algebra, which will denote $A$-valued sequences over a countable index set with summable $p$ th powers of the norms. It will always be clear from the context which we are using.

We know from Lemma 2.6 that $\ell^{2}\left(\Sigma_{1}\right)$ is not approximately amenable, and thus in this instance $\mathfrak{G}_{2}(\Sigma)$ cannot be approximately amenable ([9, Corollary 2.1]). We recall the standard fact that if two group characters are distinct, then they are non-equivalent. Hence, we have the following.

Proposition 2.10. To find a compact group for which $L^{2}(G)$ fails approximate amenability, it suffices to find such a non-abelian group with infinitely many one-dimensional representations.

EXAmple 2.11. Consider the group $G=\mathbb{T} \times S U(2)$, where $\mathbb{T}$ is the circle group and $S U(2)$ is the special unitary group of $2 \times 2$ matrices $T$ with determinant 1 such that $T^{*} T=I$. We know that $\mathbb{T}$ has dual group $\mathbb{Z}$ of countably many distinct one-dimensional unitary representations. We now extend these to the entire group $G$.

To this end, consider the representations as operators $\psi: \mathbb{T} \mapsto B(\mathbb{C})$, and extend to $\widetilde{\psi}: G \mapsto B(\mathbb{C})$ by $\widetilde{\psi}(t, M)=\psi(t)$. These are all clearly unitary and not equivalent for different $\psi \in \widehat{\mathbb{T}}$, and so there are countably many classes of the required representations. Hence our group $G$ has infinitely many classes of irreducible one-dimensional representations, and (at last) $L^{2}(\mathbb{T} \times S U(2))$ with convolution cannot be approximately amenable.

It is obvious that the pattern generalizes. 
Corollary 2.12. Suppose that $G=G_{0} \times G_{1}$ where $G_{0}$ and $G_{1}$ are compact, and $G_{0}$ is infinite and abelian. Then $L^{2}(G)$ is not approximately amenable.

Hence by choosing $G_{1}$ non-abelian, one has a range of groups for which $L^{2}(G)$ is approximately weakly amenable, but neither approximately nor weakly amenable. Note that we may even choose $G_{1}$ to be finite in this context. Recall that when $G$ is non-abelian, [8, Remark 3.2] uses any two non-commuting elements of $G$ to demonstrate the lack of weak amenability of $L^{2}(G)$.

EXAMPLE 2.13. The unitary group $U(2)$ of $2 \times 2$ matrices $T$ such that $T^{*} T=I$ has countably many one-dimensional non-equivalent unitary representations $([10, \S 29.48])$. Also, $U(2)$ is isomorphic (as a group) to the quotient group $\mathbb{T} \times S U(2) /\{(-1,-I)),(1, I)\}$ ([6, p. 146]), elucidating the existence of the one-dimensional representations. Thus $L^{2}(U(2))$ is not approximately amenable via Proposition 2.10 .

3. $\ell^{p}$-sums. Thus far, we have considered compact groups with infinitely many one-dimensional representations. A compact group is only guaranteed to have one such representation, namely the trivial one. What about compact groups with infinitely many $n$-dimensional representations? This would involve examining the space $\ell^{2}\left(M_{n}\right)$ for some fixed $n$. For the rest of this section, we will take $n$ to be some fixed natural number, and write $M(\cdot)=M_{n}(\cdot), M=M_{n}(\mathbb{C})$. We obtain an appropriate result about sequences of matrices, based on a very similar result on amenability from the recent memoir [4].

Proposition 3.1. Let $A$ be a Banach algebra. Then $A$ is approximately amenable if and only if $M_{n}(A)$ is approximately amenable.

Proof. The "if" part (which is what we really need) follows directly via the method of [4, Theorem 2.7], replacing inner derivations with approximately inner derivations. For the converse, one invokes [9, Proposition 2.3] and its subsequent remark, noting that since $M$ is finite-dimensional, the condition in the remark is satisfied.

This immediately gives us a way of untangling the approximate amenability of $\ell^{1}\left(M_{n}\right)$ algebras via the canonical Banach algebra isomorphism $\ell^{1}(M)$ $=M\left(\ell^{1}\right)$. However, a similar approach is also possible in the $\ell^{p}$ case.

Lemma 3.2. Let $1 \leq p \leq \infty$. Then

$$
\|\Gamma\|_{p}=\left(\sum_{i, j}\left|\gamma_{i j}\right|^{p}\right)^{1 / p} \quad \text { for } \Gamma=\left(\gamma_{i j}\right) \in M
$$

is an algebra norm on $M$. 
Proof. The fact that $\Gamma=0$ if and only if $\|\Gamma\|_{p}=0$, as well as the homogeneity of $\|\cdot\|_{p}$, are obvious. The triangle inequality is the same as Minkowski's inequality for $\ell^{p}$-spaces. Since $M$ is finite-dimensional, all norms on $M$ are equivalent, and hence $\|\cdot\|_{p}$ is an algebra norm.

This observation immediately leads to an important identification, whose verification is elementary.

Proposition 3.3. For $1 \leq p \leq \infty$, there is a canonical Banach algebra isomorphism from $M\left(\ell^{p}\right)$ onto $\ell^{p}(M)$.

We now give the main result of this section, discussing approximate amenability for summable sequences of finite-dimensional algebras.

TheOREM 3.4. Suppose that $\mathcal{F}$ is a finite-dimensional normed algebra. Then for $1 \leq p<\infty, \ell^{p}(\mathcal{F})$ is not approximately amenable.

Proof. Since $\mathcal{F}$ is finite-dimensional, it has a Wedderburn decomposition

$$
\mathcal{F} \approx \bigoplus_{i=1}^{N} M_{n_{i}} \odot R
$$

where $R=\operatorname{rad}(\mathcal{F})$. Hence we have the decomposition

$$
\ell^{p}(\mathcal{F}) \approx \bigoplus_{i=1}^{N} \ell^{p}\left(M_{n_{i}}\right) \odot \ell^{p}(R) \approx \bigoplus_{i=1}^{N} M_{n_{i}}\left(\ell^{p}\right) \odot \ell^{p}(R) .
$$

If $\mathcal{F}$ is not purely radical, then Proposition 3.1, Lemma 2.6 and 9, Corollary 2.1] show that $\ell^{p}(\mathcal{F})$ is not approximately amenable. On the other hand, if $\mathcal{F}$ is radical, then, being finite-dimensional, it is nilpotent, whence so is $\ell^{p}(\mathcal{F})$. Thus $\ell^{p}(\mathcal{F})$ cannot possess an approximate identity.

Remark 3.5. From the isomorphism Proposition 3.3, and Proposition 3.1, it becomes clear that $\ell^{\infty}(\mathcal{F})$ is amenable (or approximately amenable) if and only if $\mathcal{F}$ is semisimple.

Given the result obtained for finite-dimensional algebras, there is an immediate question: for $1 \leq p<\infty$, does $\ell^{p}(A)$ fail approximate amenability for every Banach algebra $A$ ? We proceed with a technical lemma.

Lemma 3.6. Suppose that $A$ is a Banach algebra, and that $I$ is a closed ideal in $A$. For $1 \leq p \leq \infty$, we have an isomorphism

$$
\ell^{p}(A) / \ell^{p}(I) \cong \ell^{p}(A / I) .
$$

Proof. Let $a=\left(a_{n}\right) \in \ell^{p}(A)$. Define $Q: \ell^{p}(A) \rightarrow \ell^{p}(A / I)$ by

$$
Q\left(a_{n}\right)=\left(a_{n}+I\right) \quad\left(a=\left(a_{n}\right) \in \ell^{p}\right) .
$$

It is immediate that $Q$ is a contractive homomorphism. 
Now suppose that $i=\left(i_{n}\right) \in \ell^{p}(I)$. Then $Q(i)=0$. If $a \in \ell^{p}(A)$ and $Q(a)=0$, then we have $a_{n} \in I$ for each $n$, and so $a \in \ell^{p}(I)$. Thus $\operatorname{ker} Q=$ $\ell^{p}(I)$.

Finally, we must check that $Q$ is surjective. Given $\left(a_{n}+I\right) \in \ell^{p}(A / I)$, we know that, for $n \in \mathbb{N}$, given $\varepsilon_{n}>0$, there is $i_{n} \in I$ such that

$$
\left\|a_{n}+i_{n}\right\| \leq\left\|a_{n}+I\right\|+\varepsilon_{n} .
$$

In particular, if $a_{n} \notin I$, then $\left\|a_{n}+I\right\| \neq 0$, and so we can choose $i_{n} \in I$ such that

$$
\left\|a_{n}+i_{n}\right\| \leq 2\left\|a_{n}+I\right\| .
$$

And if $a_{n} \in I$, then $\left\|a_{n}+I\right\|=0$, so with $i_{n}=-a_{n}$, equation (1) still holds. So for all $n$, we may choose $i_{n} \in I$ so that (1) holds.

Thus $\left(a_{n}+i_{n}\right) \in \ell^{p}(A)$ by comparison, and $Q\left(a_{n}+i_{n}\right)=\left(a_{n}+I\right)$.

Corollary 3.7. Suppose that $A$ is a Banach algebra with a closed ideal $I$ of finite codimension. Then for $1 \leq p<\infty$, $\ell^{p}(A)$ is not approximately amenable.

Proof. We immediately see that $\ell^{p}(A) / \ell^{p}(I)$ is not approximately amenable and, by [9, Corollary 2.1], $\ell^{p}(A)$ also fails approximate amenability.

Hence, $\ell^{p}(A)$ fails to be approximately amenable for many examples of Banach algebras $A$, including commutative non-radical $A$ (for then $A$ admits a non-zero character). This confirms, for example, that algebras such as $\ell^{1}\left(\ell^{\infty}\right)$ are not approximately amenable.

Coming back to compact groups, the following result is formally stronger than what we obtained previously.

Proposition 3.8. Let $G$ be a compact group with associated representation space $\Sigma$. Suppose that $G$ has infinitely many n-dimensional representations for some $n$. Then $L^{2}(G)$ is not approximately amenable.

Proof. We repeat the argument preceding Proposition 2.10, with $\Sigma_{n}=$ $\left\{\sigma \in \Sigma: \operatorname{dim} H_{\sigma}=n\right\}$ and $I=\mathfrak{G}_{2}\left(\Sigma \backslash \Sigma_{n}\right)$, to obtain

$$
\mathfrak{G}_{2}(\Sigma) / I=\ell^{2}\left(M_{n}\right)=M_{n}\left(\ell^{2}\right),
$$

which is not approximately amenable. Recall that norming $M_{n}$ under $\|\cdot\|_{\text {tr }}$ is equivalent to norming it under $\|\cdot\|_{2}$.

This enables us to examine some more groups.

Example 3.9. Consider the infinite product $G=\prod_{i=1}^{\infty} S U(2)$. It is known that $S U(2)$ has exactly one $n$-dimensional representation for each $n$ ([6, Theorem 5.39]). Thus it is easy to verify using [10, Theorem 27.43] that $G$ has only one 1-dimensional representation, but infinitely many $n$ dimensional representations for $n \geq 2$. We may now invoke Proposition 3.8 to see that $L^{2}(G)$ is not approximately amenable. 
4. Update. A new preprint [1] has very recently come to the attention of the authors. It entirely resolves the problem with the above situation, in that until now we had a result about the approximate amenability of $L^{2}(G)$ for $G$ compact only when $G$ had infinitely many $n$-dimensional representations for some fixed $n$. In [1] this issue is resolved by showing that Banach algebras $A$ which contain sequences of elements which are unbounded yet multiplier bounded fail to be approximately amenable.

Theorem 4.1 ([1, Lemma 2.4 and Theorem 2.5]). Suppose that $A$ is a Banach algebra. Suppose there exists an unbounded but multiplier bounded sequence $\left(E_{n}\right) \subset A$ such that $E_{n} E_{n+1}=E_{n+1} E_{n}=E_{n}$ for all $n$. Then $A$ fails to be approximately amenable.

This fact immediately implies that the required $\ell^{p}$-sums fail to be approximately amenable.

ExAmple 4.2 . Let $A=\ell^{p}\left(M_{n_{k}}\right)$, where at each index $k, M_{n_{k}}$ is the collection of square matrices of size $n_{k}$, normed under $\|\cdot\|_{\mathrm{tr}}$. Denoting the identity in $M_{n_{k}}$ by $I_{n_{k}}$, we see that the sequence

$$
E_{k}=\left(I_{n_{1}}, \ldots, I_{n_{k}}, 0,0, \ldots\right)
$$

satisfies the criteria of Theorem 4.1. Hence, $A$ fails to be approximately amenable.

REMARK 4.3. Interestingly, this example seems to be at the heart of [1. Example 2.7], however their hypothesis that $\left\|I_{n_{k}}\right\| \rightarrow \infty$ (which thus appears to be superfluous) compensates for the fact that they do not take sums of the $I_{n_{k}}$. The same result will follow for $\ell^{p}\left(A_{n}\right)$ for any sequence $\left(A_{n}\right)$ of Banach algebras each of which contains a non-zero idempotent. However, the method of Theorem 4.1 and Example 4.2 does not apply, for instance, to the situation where all the $A_{n}$ equal a fixed finite-dimensional radical algebra, which is covered by Theorem 3.4 .

THEOREM 4.4. For any infinite compact group $G$, the algebra $L^{2}(G)$ fails to be approximately amenable.

EXAMPLE 4.5. Consider the compact non-abelian group $S U(2)$. It has exactly one $n$-dimensional representation for each $n \in \mathbb{N}$ ([6] Theorem 5.39). We have the group isomorphisms ([10, Theorem 29.36 and $\S 29.49])$

$$
S O(3) \approx S U(2) /\{I,-I\} \text { and } O(3) \approx\{-1,1\} \times S O(3) .
$$

The fact that the $L^{2}$-algebras of these groups fail approximate amenability is encapsulated by the new result above.

Acknowledgements. This paper is based on part of the first author's $\mathrm{PhD}$ thesis at the Australian National University. 


\section{References}

[1] Y. Choi and F. Ghahramani, Approximate amenability of Schatten classes, Lipschitz algebras and second duals of Fourier algebras, Quart. J. Math. (2010), doi:10.1093/ qmath/hap034.

[2] Y. Choi, F. Ghahramani and Y. Zhang, Approximate and pseudo-amenability of various classes of Banach algebras, J. Funct. Anal. 256 (2009), 3158-3191.

[3] H. G. Dales, Banach Algebras and Automatic Continuity, London Math. Soc. Monogr. 24, Clarendon Press, New York, 2000.

[4] H. G. Dales, A. T.-M. Lau and D. Strauss, Banach algebras on semigroups and their compactifications, Mem. Amer. Math. Soc., to appear.

[5] H. G. Dales, R. J. Loy and Y. Zhang, Approximate amenability for Banach sequence algebras, Studia Math. 177 (2006), 81-96.

[6] G. B. Folland, A Course in Abstract Harmonic Analysis, Stud. Adv. Math., CRC Press, Boca Raton, FL, 1995.

[7] F. Ghahramani and A. T.-M. Lau, Approximate weak amenability, derivations and Arens regularity of Segal algebras, Studia Math. 169 (2005), 189-205.

[8] - - - Weak amenability of certain classes of Banach algebras without bounded approximate identities, Math. Proc. Cambridge Philos. Soc. 133 (2002), 357-371.

[9] F. Ghahramani and R. J. Loy, Generalized notions of amenability. J. Funct. Anal. 208 (2004), 229-260.

[10] E. Hewitt and K. A. Ross, Abstract Harmonic Analysis. Vol. II, Grundlehren Math. Wiss. 152, Springer, New York, 1970.

P. Bharucha, R. J. Loy

Mathematical Sciences Institute

Australian National University

Canberra, ACT 0200, Australia

E-mail: pourus@gmail.com

rick.loy@anu.edu.au 